\title{
Especies locales, mercado y transporte en las investigaciones embriológicas: el estudio de la poliembrionía en armadillos a principios del siglo $\mathrm{XX}$
}

\section{Local species, the market, and transportation in embryological research: the study of polyembryony in armadillos in the early twentieth century}

\footnotetext{
Susana V. García

Investigadora del Consejo Nacional de Investigaciones Científicas y Técnicas (Conicet)

Archivo Histórico del Museo de Ciencias Naturales/

Universidad Nacional de La Plata

Paseo del Bosque $s / n$

1900 La Plata - Buenos Aires - Argentina

garcia_su@yahoo.com.ar

Recibido para publicación en septiembre 2007.

Aprobado para publicación en marzo 2008.
}

GARCÍA, Susana V. Especies locales, mercado y transporte en las investigaciones embriológicas: el estudio de la poliembrionía en armadillos a principios del siglo XX. História, Ciências, Saúde - Manguinhos, Rio de Janeiro, v.15, n.3, p.697-717, jul.-set. 2008.

\section{Resumen}

Se examina en un caso concreto la afirmación general de la importancia de las redes de comunicación y la movilidad de las cosas para la ciencia. De qué forma esto jugó un papel relevante en las investigaciones sobre la embriología de ciertas especies de armadillos a principios del siglo XX? Como intentamos mostrar, el acceso a las hembras preñadas de esas especies silvestres y los tiempos en el transporte hasta el laboratorio fueron cuestiones substanciales en los inicios de esas investigaciones. Las estrategias para obtener muestras en el campo y la temporalidad de ciertos fenómenos, los circuitos comerciales en los que participan los organismos estudiados y su trasformación en el laboratorio permiten vislumbrar aspectos de cómo trabajaron y qué problemas enfrentaron esos científicos.

Palabras-clave: historia de la embriología; armadillos; prácticas científicas; poliembrionía.

\section{Abstract}

The article examines the general affirmation that communication networks and mobility are important to science by examining how these factors played a relevant role in one specific case, that is, research on the embryology of certain species of armadillos in the early twentieth century. As we show, access to pregnant females of the species and transport time from the wild to the laboratory were significant issues in the early days of such research. The strategies used to obtain field samples, the temporality of certain phenomena, the commercial circuits of which the organisms under study were a part, and the transformations they underwent in the laboratory all afford a glimpse at some aspects of how these scientists approached their work and what problems they faced in producing new knowledge.

Keywords: history of embryology, armadillos, scientific practices, polyembryony. 
$\mathrm{E}$ $\mathrm{n}$ los últimos años, los historiadores de las ciencias han comenzado a mostrar el espectro de interacciones entre los saberes científicos y las infraestructuras del transporte y la comunicación. Las mismas pueden extenderse desde las ventajas prácticas para la circulación de información, objetos y personas, las facilidades para acceder a sitios distantes, la movilidad de equipamiento, provisiones y personal para el trabajo de campo así como la cantidad y los tipos de muestras traídas de los lugares estudiados (Podgorny, 2002, 2005). Los medios de transporte fueron un elemento crucial para el trabajo de campo no solo en relación a esas posibilidades, sino también en la medida en que la construcción y tendido de las rutas y vías férreas abrieron un corredor para la observación de la naturaleza y su constitución geológica, tal como se ha mostrado en el caso del ferrocarril (Vetter, 2004; Freeman, 2001; Moore, 1986). También lo fueron para los científicos que trabajaban en los laboratorios y gabinetes urbanos, ya sea para acceder a sus objetos de estudio, para el envío de instrucciones a los trabajadores en el campo, así como para el intercambio de suministros e información. Otro tipo de interacciones incluyeron relaciones más complejas como las redes de colaboración y los intercambios recíprocos de favores entre los científicos y el personal de las empresas del transporte y la comunicación. Los puertos, estaciones de ferrocarril o estafetas postales o telegráficas fueron sitios de circulación de información y cosas, pero también puntos desde donde se remitieron, y en muchos casos se conformaron, colecciones y datos para los museos y otras instituciones científicas. Recordemos, por ejemplo, la instalación de estaciones meteorológicas en oficinas de Correos y Telégrafos de distintos puntos del territorio argentino coordinados por la Oficina Meteorológica de Córdoba a fines del siglo XIX (Bose, 1966).

Por otro lado, esos proyectos tecnológicos de dominio del territorio a través del establecimiento de vías de comunicación y de movilidad de cosas y personas apelaron a saberes científicos, promoviendo exploraciones y estudios para seleccionar las rutas más convenientes. Asimismo, se ha reconocido cómo los modos particulares de viajar pudieron guiar y conformar las experiencias de la naturaleza y, al mismo tiempo, contribuir a la construcción del espacio por donde se transitaba (Sorrenson, 1996; Vetter, 2004; Lopes, 2001). En ese sentido, los medios de transporte pueden ser considerados como dispositivos que afectan cómo los viajeros-usuarios perciben y construyen el espacio alrededor de ellos. Paralelamente, también fueron un agente de trasformación económica, abriendo y conectando nuevas regiones y productos en el circuito de los mercados nacionales o internacionales. En muchos casos, los especimenes de estudio y objetos de investigación adquirieron visibilidad o fueron accesibles para los científicos en estrecha relación con los mercados urbanos de frutas y carnes y las rutas comerciales. En el campo, los cazadores, observadores locales o guías indígenas también desempeñarían un papel importante en ello, así como en los primeros informes sobre los ciclos de vida y hábitos de ciertos animales.

En este trabajo se busca examinar, en un caso concreto, la afirmación general de la importancia de las redes de comunicación, los medios de transporte y la movilidad de las cosas para la ciencia. En particular se procura explorar de qué forma esto jugó un papel relevante en la producción de conocimiento sobre la reproducción y la embriología de especies americanas no domesticadas ni domesticables para la época, como ciertos tipos de armadillos. 
En los inicios del siglo XX, dos especies relacionadas de estos mamíferos, la llamada popularmente mulita (Dasypus hybridus) y el armadillo de nueve bandas (Dasypus novemcinctus), fueron objeto de investigaciones sistemáticas en torno a las peculiaridades que presentaba su desarrollo embriológico. Dos grupos de investigadores, uno ubicado en la Universidad de La Plata (Argentina) y otro en la Universidad de Tejas en Austin (Estados Unidos), trabajaron simultáneamente sobre el mismo fenómeno, aunque de forma independiente y con las especies regionales. Como intentamos mostrar en este trabajo, el acceso a las hembras preñadas de estos animales silvestres y los tiempos en el transporte hasta el laboratorio fueron cuestiones substanciales en los inicios de estas investigaciones. Vinculado con ello, en las siguientes páginas se procura mostrar algunos aspectos de las prácticas del trabajo embriológico, así como la interrelación entre especies locales, redes de transporte y mercados y la temporalidad tanto de los organismos como en el trabajo de los científicos.

\section{Prácticas embriológicas y observaciones en armadillos}

En el último cuarto del siglo XIX, la observación y comparación de las placentas de diferentes grupos de mamíferos fue cobrando importancia, tanto que algunos naturalistas propusieron la clasificación de los mamíferos monodelfos (placentarios) de acuerdo a este carácter, mientras que la embriología fue considerada uno de los pilares para establecer relaciones filogenéticas junto con la anatomía comparada de las formas adultas (Bowler, 1996; Benson, 1981). Los primeros informes sobre las placentas observadas en una o dos hembras preñadas mostraron una gran variabilidad en el caso de los edentados, grupo de animales agrupados por el carácter de su dentadura, integrado por los armadillos, perezosos y otras formas vivientes y fósiles característicos de Sudamérica. También dentro de la familia de los armadillos se observaron algunos tipos diferentes de placenta. Estos animales se diferencian del resto de los mamíferos vivientes por tener el dorso del cuerpo cubierto por un caparazón óseo y placas móviles, mientras que por las características de su dentición fueron clasificados dentro del grupo de los edentados. En general, los armadillos fueron considerados como un grupo peculiar de mamíferos arcaicos, especialmente después del viaje de Darwin en el Beagle. Médicos, viajeros, ingenieros y naturalistas fueron remitiendo a museos y a anatomistas europeos observaciones y restos de esqueletos, caparazones, pieles o ejemplares conservados en alcohol. Los restos fósiles y ciertas especies vivientes atraerían la atención de diferentes naturalistas europeos y locales.

Algunos científicos europeos pudieron examinar uno o dos úteros conservados del armadillo de nueve bandas (Dasypus novemcinctus), una especie con una amplia distribución entre el norte de la Argentina y México y que se extendió por el estado de Tejas a mediados del siglo XIX (Smith, Douhty, 1984). Encontraron un curioso fenómeno en las membranas embrionarias: los fetos, en número de cuatro, poseían cada uno un amnios propio, pero las placentas estaban unidas y los cuatros fetos estaban rodeados por un corion común, cuando en general era conocido que cada embrión de mamífero poseía su propio corion. En 1878, el naturalista francés Alphonse Milne-Edwards (1835-1900), profesor del Museo de Historia Natural de París, al examinar un ejemplar conservado en alcohol, observó este 
fenómeno y, luego, en otra vesícula embriológica más avanzada. En relación con ello, enumeró tres posibles explicaciones: (a) la vesícula de Graaf contenía varios huevos en vez de uno, (b) los cuatro huevos fecundados estaban incluidos en un depósito plasmático formado por las paredes del útero o del oviducto o (c) los corions de los cuatro embriones se habrían fusionado, inclinándose por esta última posibilidad (Milne-Edwards, 1879).

$\mathrm{Al}$ año siguiente, una observación similar fue hecha por el médico y naturalista francés radicado en México, Alfred Dugès (1826-1910), quien en una estancia de campo pudo examinar el interior de una hembra preñada capturada por unos obreros, tras lo cual remitió a MilneEdwards un informe sobre ello y publicó una breve noticia (Dugès, 1881, 1902). Poco antes, también el zoólogo Rudolf Albert von Kölliker (1817-1905) tuvo ocasión de observar un útero con cuatro embriones de la misma especie y comentó esto en la segunda edición de su tratado de embriología (Kölliker, 1882). Diferentes explicaciones fueron elaboradas por los anatomistas para esa aparente 'anomalía' de un corion rodeando varios fetos. Una de las explicaciones más populares, aunque hipotética ya que las etapas más tempranas en el desarrollo del armadillo y en general su embriología eran desconocidas, era que inicialmente cada embrión tenía su corion pero, durante el desarrollo, las paredes adyacentes de los corions primitivos se fusionaban entre ellos, formando uno solo en común.

Por otro lado, en Sudamérica existía una creencia popular que ciertas especies de armadillos como las llamadas mulita y la Peba, o tatú negro (nombre regional del armadillo de nueve bandas), tenían en cada parición varias crías y todas del mismo sexo. Esta curiosa información fue registrada por el ingeniero español Félix de Azara (1742-1821) durante su estadía en el Paraguay y la región del Plata para la medición del límite entre los dominios del imperio español y portugués al terminar el siglo XVIII. Azara (1801) describió diversas especies de armadillos, sus hábitos y los usos dados por los pobladores locales. Entre ello, se interesó por comprobar la idea de que todas las crías de la mulita eran del mismo sexo, lo que pudo observar personalmente al abrir el útero de dos hembras preñadas en estado avanzado.

El mismo comentario fue repetido varias décadas después por el naturalista alemán Hermann Burmeister (1807-1892), director del Museo Público de Buenos Aires, donde se conservaba un ejemplar de mulita con nueve fetos muy desarrollados y todos del mismo sexo. Esto también le sería confirmado por personas que habían vivido en el campo (Burmeister, 1876). A mediados de la década de 1880, el naturalista alemán Hermann von Ihering (1850-1930), por entonces empleado del Museo de Río de Janeiro, publicó unas notas al respecto (Ihering, 1885, 1886a, 1886b). Al parecer, una creencia similar existía entre nativos de Brasil y se interesó por cotejar científicamente lo que hasta ese momento aparecía como una pieza interesante del folclore local. Ihering examinó dos hembras preñadas de armadillo remitidas desde Paraguay, cada una de las cuales tenía ochos fetos encerrados dentro de un mismo corion. En ambos casos, todos los fetos eran machos. En base a la conformación de las membranas embrionarias y por ser los embriones del mismo sexo, postuló la hipótesis de que todos los embriones de cada grupo eran el producto de la escisión de un solo huevo fecundado en un cierto número de primordios embrionarios separados. Señaló, además, que este fenómeno podría considerarse como un caso de generación alternante (Generationswechel) en mamíferos (Ihering, 1886a). Aunque esto 
último sería cuestionado por algunos investigadores europeos por la falta de mayores datos sobre el desarrollo embriológico de estos animales y en el marco de una serie de debates sobre el concepto de Generationswechel (Farley, 1982). Ihering aparentemente no profundizó más en este fenómeno y en los siguientes años tampoco se registran investigaciones al respecto, aunque el tema volverá a cobrar interés en los primeros años del siglo XX.

En el marco de los estudios sobre los gemelos humanos, una hipótesis diferente fue dada en 1901 por el investigador polaco Aleksander Rosner (1867-1930) sobre la base de un estudio histológico de los ovarios de un ejemplar de Dasypus novemcinctus. Los órganos genitales femeninos de dos animales le habían sido remitidos por Ihering, pero sólo uno de los especimenes estaba suficientemente bien preservado para admitir un examen histológico. La disección del otro par de ovarios mostró que los folículos contenían varios huevos, algunos con cuatro huevos, número que coincidía con el de los individuos de una camada. Sobre estas observaciones, Rosner concluyó que los cuatro embriones que típicamente formaban la camada del armadillo de nueve bandas provenían no de un sólo huevo sino de los cuatro huevos encerrados en un folículo, los cuales eran liberados simultáneamente hacia el útero donde serían fecundados y posteriormente las paredes continuas de los cuatro embriones se fusionarían, conformando un corion común. Los estudios posteriores de otros investigadores mostraron que el material observado por Rosner era patológico o excepcional, ya que no se encontraron las condiciones del ovario del armadillo por él descriptas y era muy extraordinario encontrar más de un huevo en un folículo (Newman, Patterson, 1910; Newman, 1922). Esto remite a algunos problemas interesantes en la historia de las ciencias. Por un lado, cómo se determina la normalidad de un proceso, situación u observación o cómo se otorga credibilidad a los datos observados cuando no se repite la observación o cuando resulta casi imposible. ${ }^{1}$

Por otro lado, esto se vincula con el problema de la obtención y circulación de muestras convenientemente preparadas y conservadas para determinados estudios, como por ejemplo, para la histología y anatomía. Recordemos que los manuales para los naturalistas viajeros, pero también los textos más específicos de embriología e histología, indicaban los elementos y procedimientos necesarios para la conservación y transporte de los ejemplares a los centros de estudio durante los viajes y de acuerdo al tiempo que pudiera transcurrir antes de su adecuada preparación y estudio en el laboratorio. Los medios técnicos por los cuales se podían hacer de los animales, o partes de ellos, objetos móviles y 'estables' para la ciencia (Latour, 1992) abarcaban: operaciones de preparación y extracción de líquidos y contenido estomacal, mediciones, registro de los colores y dibujos de los ejemplares frescos así como importantes cantidades de alcohol de buena calidad para su preservación. Posteriormente se multiplicaría la oferta de sustancias conservantes y fijadoras y las manipulaciones necesarias para el endurecimiento de las estructuras a observar y su preservación durante el viaje del campo al laboratorio así como su registro fotográfico. No obstante, estas técnicas y procedimientos que intervendrían en la movilidad y visualización de los objetos científicos es un tópico aún poco explorado en la historia de las prácticas de las ciencias biológicas.

En la primera década del siglo XX, la cuestión de la poliembrionía (la formación de varios embriones a partir de un solo huevo) adquirió un gran interés científico. Este 
fenómeno fue observado en diferentes grupos de animales: parásitos, insectos, briozoos, tunicados y por medio de intervención experimental, en equinodermos y peces. En ese marco, el proceso de la poliembrionía cobraría una nueva significación en relación con los casos de blastotomía experimental registrados por algunos investigadores en la década de 1890. Otra de las cuestiones vinculadas con ese fenómeno, fue si, por ejemplo entre los insectos, la poliembrionía procedía o seguía filogenéticamente a otros modos de reproducción. Asimismo, el interés científico por ese fenómeno también se vinculó al gran debate sobre la determinación de sexo (Maienschein, 1984). Los estudios publicados sobre la poliembrionía y la observación que todos los individuos de una camada eran del mismo sexo parecía confirmar un hecho largamente sospechado pero incompletamente demostrado hasta ese momento: el conocimiento que la determinación del sexo dentro del óvulo fertilizado estaba definitivamente fijado antes de la primera segmentación de su núcleo (Bugnion, 1907). De acuerdo con esto, algunos autores como el zoólogo francés Lucien Cuénot (1866-1951), quien había buscado mostrar experimentalmente que el sexo no estaba influenciado por las condiciones exteriores y pensaba que la determinación tenía lugar tan tempranamente como en el estado de huevo, señaló que seguramente los armadillos de una misma camada eran verdaderos gemelos. En este marco, algunos autores reabrieron la cuestión acerca de la poliembrionía en estos animales, reconociendo que con toda probabilidad, los múltiples embriones procedían de un mismo huevo.

En 1909 comienzan a publicarse los resultados de dos investigaciones sobre este tema, desarrolladas simultáneamente aunque de forma totalmente independiente por dos grupos de científicos americanos. Mientras en la Argentina, Miguel Fernández (1882-1950), zoólogo entrenado en las universidades de lengua alemana, estudiaba este fenómeno en la mulita; en Estados Unidos dos investigadores, Horatio Hackett Newman (1875-1957) y John Thomas Patterson (1878-1960), en la Universidad de Tejas, en Austin, analizaban el caso del armadillo de nueve bandas de Tejas. Estos trabajos no estaban basados en la observación casual de

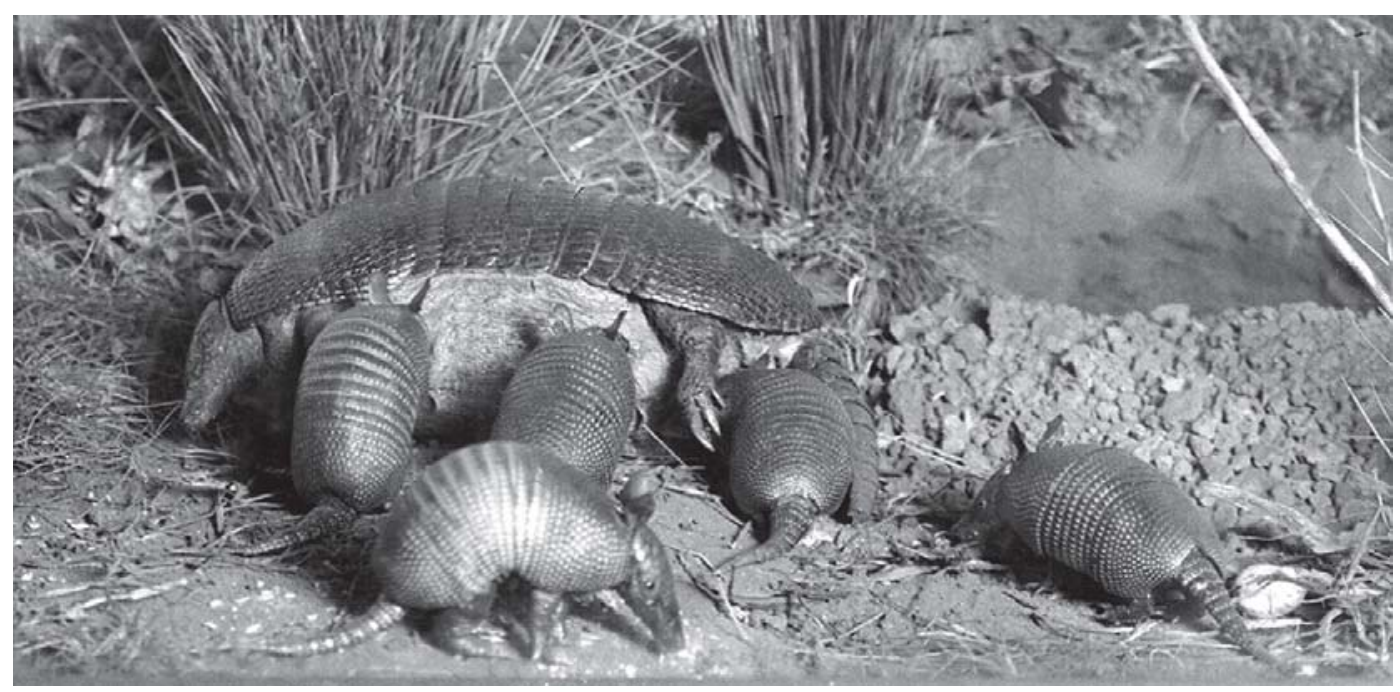

Figura 1 - Una mulita con sus crías (Archivo Histórico del Museo de La Plata) 
uno o dos úteros, sino en investigaciones sistemáticas sobre el desarrollo embriológico de esos animales. Para esto, un gran número de hembras preñadas serían requeridas para lograr resultados significativos, especialmente para poder fijar los estadios más tempranos de ese proceso y realizar disecciones utilizables como evidencia. Esto era más problemático en el caso de los mamíferos no domésticos y en aquellas especies que sólo se reproducían una vez al año como la mulita y el armadillo de nueve bandas.

Una de las principales dificultades relacionada con las prácticas embriológicas era obtener una serie completa de muestras de las sucesivas etapas del desarrollo, algo que había sido dificultoso aún en los clásicos trabajos con el pollo, mientras que en los mamíferos esto podía llegar a ser extremadamente costoso y en algunos casos prácticamente imposible. En estos animales, el estudio del desarrollo embrionario se complicaba por la relación del feto con la madre y la invisibilidad de ese proceso. En ese sentido, el cuerpo de las hembras preñadas se presentaba como un sólido opaco, donde el crecimiento de las estructuras embriológicas no podía ser observado directamente. Esto era inferido luego de que el crecimiento había tenido lugar, deduciendo o interpretando que había sucedido entre dos cortes secuenciales de momentos preservados o fijados en el tiempo. El desarrollo era tratado como una serie de "eventos de desarrollo", donde un evento era un cambio discreto en las células o los tejidos analizados. Los diferentes momentos o etapas eran registrados y ordenados a partir de una serie de cortes preparados y observaciones obtenidas de diferentes individuos de la especie estudiada. Para cada momento o etapa del desarrollo, un nuevo organismo era matado y cortado para mirar en su interior un punto particular de tiempo. En ese sentido, el crecimiento se inducía de manera independiente al espacio y tiempo concreto en el que se desarrollaba el proceso estudiado. Como ha señalado Hopwood (1999), el desarrollo secuencial del proceso ontogenético era una composición tanto temporal como espacial. Los preparados histológicos, las microfotografías, los dibujos y esquemas hablaban de la habilidad de los investigadores para capturar los momentos discretos en un proceso continuo, pero también de la disponibilidad de los medios técnicos y los organismos de estudio.

En los animales que no presentaban dificultades para su crianza y reproducción en cautiverio, y mientras se contara con una gran cantidad, la cronología del desarrollo podía reconstruirse bastante minuciosamente tomando como punto de inicio el momento del coito. Así, por ejemplo, en un estudio de los primeros estadios embriológicos del cobayo, una, dos o a veces más hembras fueron matadas por cada hora trascurrida después de la fecundación, a fin de poder reconstruir el desarrollo durante las primeras 170 horas (Lams, 1913). Obviamente, esto implicó el sacrificio de una gran cantidad de ejemplares para la extracción de sus ovarios y úteros, los cuales eran sumergidos inmediatamente en los líquidos fijadores y luego cortados, teñidos, observados en el microscopio, dibujados y descriptos.

Las técnicas histológicas de matar, fijar, seccionar, teñir selectivamente determinado grupos de células y preparar para su observación microscópica y su registro gráfico y visual estuvieron en el centro de la delineación de las complejas estructuras de los organismos vivientes en los inicios del siglo XX. Todo un conjunto de dispositivos técnicos, visuales y teóricos se pondría en juego para superar la doble invisibilidad de lo pequeño y lo homogéneo. El perfeccionamiento de esos métodos - coloración, inclusión en parafina, 
práctica de finos cortes que se montan en portaobjetos y reconstrucción tridimensional ocuparon la atención de gran cantidad de embriólogos. El biólogo norteamericano Thomas Morgan (1941, p.13-14), cuestionando el auge de la embriología descriptiva en la transición del siglo, recordaba:

La tentación era irresistible, especialmente cuando se creía que lo que se estaba viendo y describiendo era la historia de la creación, o como se decía, la evolución. Pronto se dispuso de un inmenso material referente al desarrollo de todas las principales formas animales, y bellas y numerosas ilustraciones aparecieron en un cúmulo de monografías. Cuando más perfecto era el artista, más brillante era su obra. La anatomía del desarrollo llegó a ser tan exactamente conocida como lo era la anatomía de las estructuras adultas, la cual había exigido igualmente una cuidadosa observación y un sentido artístico del color y la perspectiva.

Precisamente, los atlas, los modelos y las imágenes de estadios embriológicos de otras especies funcionaban como herramientas de laboratorio en tanto permitían las comparaciones, ayudaban a ordenar los cortes o a completar la cronología del desarrollo 'normal'. Esto ha sido examinado por Nick Hopwood $(2005,2007)$ en el caso de las series de láminas editadas por el alemán Franz Keibel, en las cuales se describían embriones de varios vertebrados sobre la base de una serie estandarizada. Sus autores recolectaron diversos objetos y los analizaron a partir de un marco común, una precondición para el trabajo comparativo. Las imágenes difirieron debido a que los embriólogos pudieron estudiar las especies más libremente que otros, aunque en cierto nivel todos compartieron un proyecto común. A través de ellos, como menciona Hopwood, parece factible mapear un "imperio embriológico". Los embriólogos y zoólogos fueron incentivados a analizar especies seleccionadas por sus posiciones sistemáticas y/o porque sus vínculos con las actividades agropecuarias, caza, pesca o turismo podían facilitar el acceder a ellos.

Los investigadores pudieron obtener animales a partir de la colaboración o la compra a granjeros, cazadores o pescadores o ir ellos mismos al campo para obtener ejemplares frescos. Las estaciones o laboratorios marinos, como el famoso de Nápoles creado en 1872, se convirtieron en los sitios claves para los estudios embriológicos y anatómicos de los organismos marinos. Allí, todos los días los investigadores podrían contar en sus mesas de trabajo con ejemplares frescos provistos por los pescadores o los acuarios del establecimiento, además de disponer de otras herramientas de trabajo como instrumental y bibliografía (Ghiselin, Groeben, 1997). Otros embriólogos usaron las redes imperiales para traer a los laboratorios europeos 'fósiles vivientes' o realizar excursiones para estudiar aquellas especies consideradas más primitivas o de transición entre dos grupos con un rango de distribución fuera de Europa, como por ejemplo, los marsupiales y monotremas de Australia ${ }^{2}$, los prosimios de Indonesia o los peces pulmonados del Chaco paraguayo (Macleod, 1994; Hopwood, 2007).

Ligado a este tipo de investigaciones aparecía el problema de obtener un número suficiente de ejemplares para su estudio en el laboratorio y especialmente el de lograr fijar los estadios más tempranos en el desarrollo embriológico, un tema que cobró especial interés en los inicios del siglo XX en el marco de los debates en torno a la teoría de la gastrulación y su aplicación en los mamíferos. Un problema, a su vez, vinculado con la estacionalidad de los animales, el período de gestación, las estrategias para conseguir ejemplares vivos y preñados así como la posibilidad de obtener una secuencia de estadios embrionarios jóvenes y realizar disecciones 
utilizables como evidencia. En el caso de los investigadores americanos que trabajaron con las especies locales de armadillos, como analizaremos a continuación, también debieron articular redes de comunicación y provisión de ejemplares preñados y vivos (o frescos) desde el campo. En ello, los tiempos de transporte de los animales hasta el laboratorio sería un factor decisivo para fijar los primeros momentos del desarrollo en esas especies silvestres.

\section{La confirmación de la poliembrionía 'específica' en la mulita}

Una prueba definitiva sobre la suposición de la "poliembrionía específica" entre los armadillos fue aportada por Miguel Fernández en 1909. Este zoólogo de nacionalidad argentina-alemana, era hijo de padre argentino y madre alemana, había vivido su infancia en una propiedad rural en la provincia de Buenos Aires, antes de que su familia se instalara en Montevideo (Cordero, 1950; Birabén, 1951). Realizó sus estudios universitarios en Zürich, primero en la Escuela Superior Politécnica donde se inclinó por las ciencias químicas, y luego siguió los cursos de zoología y anatomía comparada en la Universidad. Allí trabajó en el laboratorio de Arnold Lang (1855-1914), un destacado morfólogo que había sido

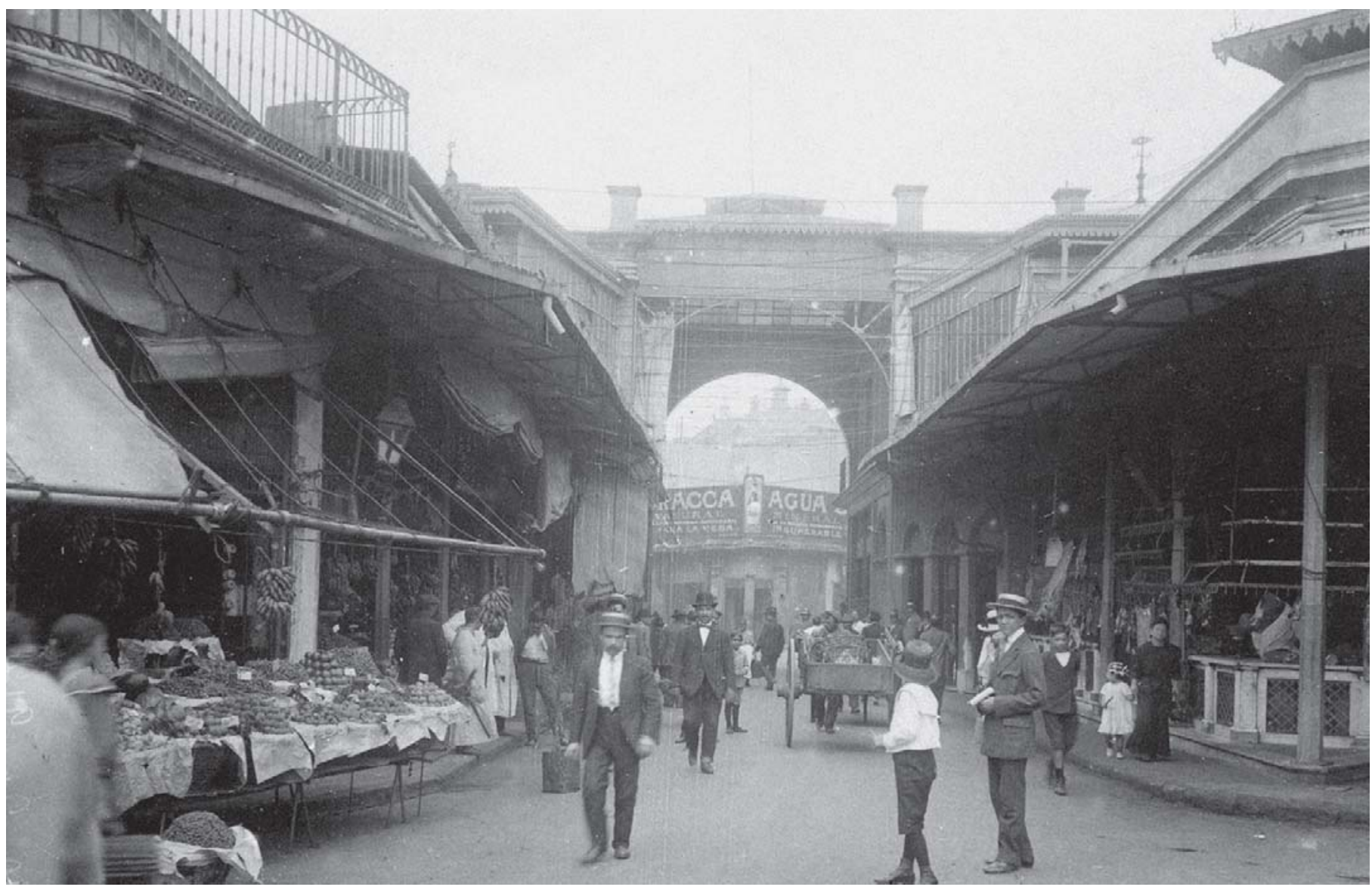

Figura 2 - Interior de un mercado de Buenos Aires a principios del siglo XX (Archivo General de la Nación) 
alumno y colaborador de Karl Vogt en Ginebra, Anton Dohrn en la Estación Marina de Nápoles y Ernst Haeckel en Jena (Nyhart, 1995; Bowler, 1996). En 1904-1905, Fernández defendió su tesis sobre el origen y la anatomía del aparato vascular de tunicados y luego complementaría su formación en otras instituciones científicas europeas: asistió un semestre a la Universidad de Heidelberg y al igual que otros zoólogos de la época, realizó algunas estadías en un laboratorio marino. A principios de 1906, con 23 años, una acreditada formación científica y con la recomendación de su director de tesis, este investigador llegó a La Plata convocado como profesor a cargo de los cursos de zoología en la nueva Facultad de Ciencias Naturales organizada en el contexto del Museo de La Plata (García, 2004).

La contratación de Fernández se habría operado, al igual que en otros casos, a través de las redes e intermediación de otros científicos extranjeros residentes en el país. Destaquemos que la incorporación de profesores e investigadores europeos y norteamericanos a las instituciones científicas argentinas formó parte de las políticas de modernización cultural y científica del país en distintos momentos de su historia, como ha sido mencionado ampliamente en la historiografía de las ciencias argentinas. En la última parte del siglo XIX y gran parte del XX, se presenció la contratación y llegada de profesores de lengua alemana que se integrarían a los establecimientos científicos argentinos, estableciendo en muchos casos vínculos entre ellos, redes de negociación locales y un sentimiento de pertenencia a una comunidad académica internacional. Si bien, frecuentemente se los ha reunido a todos bajo el arquetipo de una comunidad o un grupo unido por una misma adscripción lingüística, el análisis de las trayectorias y la actuación de esos científicos extranjeros radicados en la Argentina revela tanto lealtades y alianzas cruzadas, como diversas tendencias ideológicas además de orígenes, formación e intereses variados (García, Podgorny, 2000). En el caso de Fernández, cabe mencionar que las prácticas científicas promovidas por este investigador aparecerían como un estilo de trabajo diferente a lo que habitualmente se venía realizando en el espacio de los museos argentinos (Podgorny, 2000) y en general en el campo de la zoología en la Argentina.

Desde su llegada a La Plata, a principios de 1906, empezó a reunir ejemplares de diferentes especies de armadillos para estudiar su embriología, algunos aspectos morfológicos y establecer posibles relaciones filogenéticas. Asimismo, recibiría datos y ejemplares de mulitas y peludos para sus estudios a través de algunos colaboradores en el campo, principalmente de origen alemán o que compartían esta lengua. Fernández reconoció que la razón principal por la que se concentró inicialmente en el caso de la mulita, una de las formas de armadillo más frecuente en la provincia de Buenos Aires, fue el fácil acceso a ejemplares vivos de esta especie, ya que su carne era muy apreciada y vendida en los mercados urbanos (Fernández, 1915a). Además, el período anual de caza coincidía con la época de preñez de la mulita y particularmente durante las fases tempranas de la gestación en los meses de mayo, junio y julio, gracias a lo cual Fernández pudo seleccionar muchas hembras vivas y preñadas entre los miles que se vendían anualmente a los mercados urbanos.

En los campos de la provincia de Buenos Aires, tanto la caza de la mulita como la del peludo era un pasatiempo popular: "la manera de cazarlos (como su carne es muy apetecida) es bien simple. En las noches de luna, los habitantes de la campaña suelen salir á peludear, argentinismo que expresa la cacería del Peludo. Ella se ejecuta por medio de perros, ó 
dando al animal un golpe con el mango del rebenque ó con un palo. La Mulita se caza de día" (Holmberg, 1898, p.491). Aunque la carne de estas dos especies de armadillos era consumida en el campo, sólo la de la mulita era apreciada en los círculos urbanos. Como describió el naturalista argentino Eduardo Holmberg (1898, p.492):

\footnotetext{
de toda la Familia á la que pertenece, es la especie más perseguida, y con razón, porque como fiambre, difícilmente podrá encontrarse (en la República Argentina) nada más delicado. Los que saben comerla la prefieren hervida y fría, pues caliente es en extremo empalagosa é indigesta, sobre todo si está muy gorda. Los cordon-bleu de Buenos Ayres, particularmente franceses, la condimentan demasiado en los hoteles. No es carne la suya para enfermos, ni para convalecientes, ni para niños de ciudad, y dividida en cuatro, dá ración para cuatro personas sanas. Solo, es manjar que excluye el vino ...
}

Como señala este naturalista, en la transición del siglo el consumo de mulita pareció estar de moda en los hoteles y restaurante de Buenos Aires, mientras que algunos folletos de propaganda recomendaban al viajero no dejar de probar este 'manjar' regional. Las estadísticas oficiales también registraron un incremento constante, aunque fluctuante, en la comercialización anual de mulitas durante esos años: mientras en el año 1890, 7.587 mulitas llegaron a los mercados de Buenos Aires, una década después se registraron 26.154 animales. Cabe señalar que en el boletín mensual de estadística municipal de la ciudad de Buenos Aires, también se registra un movimiento similar con respecto al consumo de otras especies silvestres como patos y perdices, así como la incorporación de nuevos productos como ostras y la llegada de pescado de la costa atlántica gracias a la extensión del ferrocarril a Mar del Plata hacia 1886. De hecho, las estadísticas compiladas por el jefe de esa estación

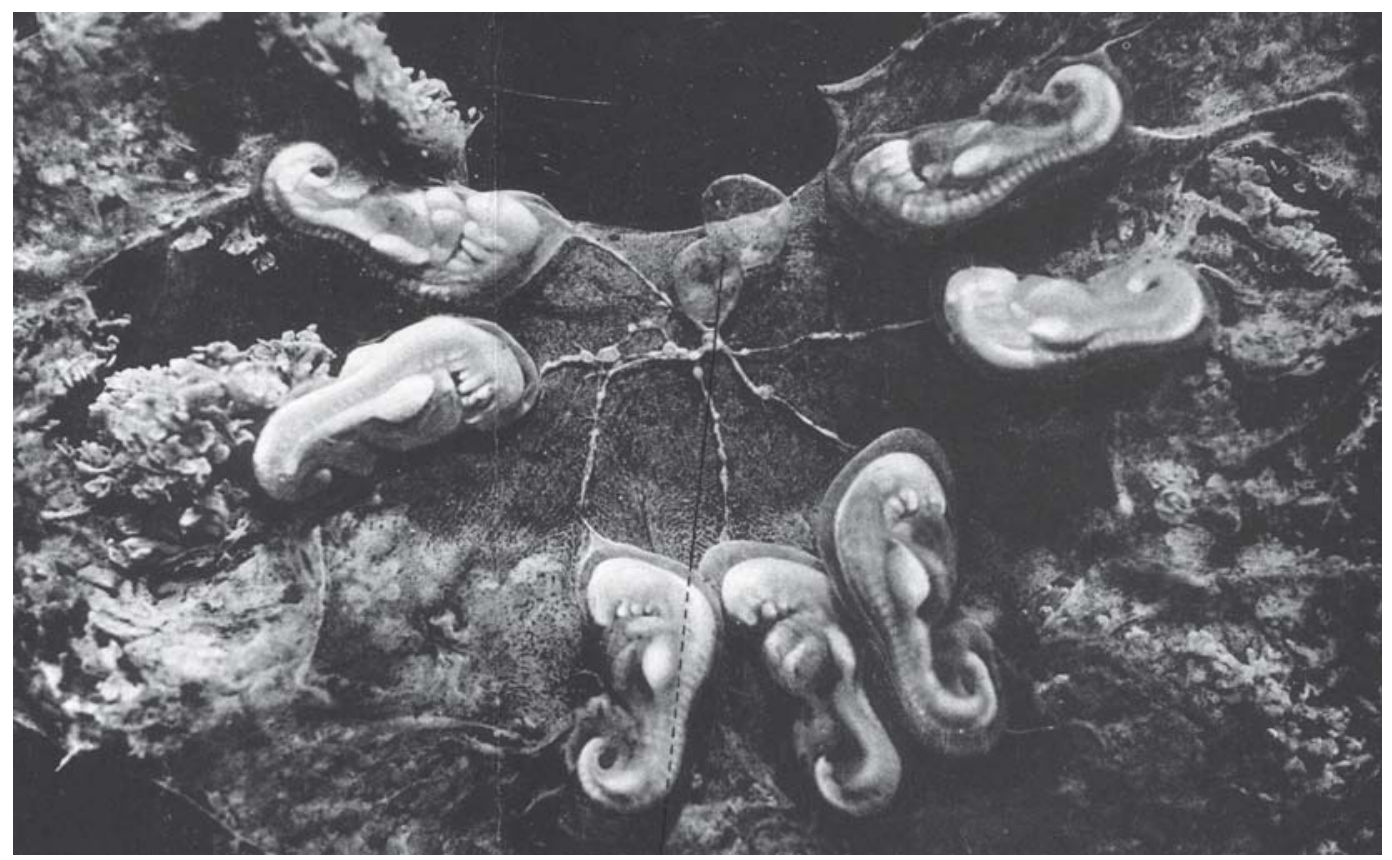

Figura 3 - Vista del lado interno de una vésicula embrionaria extendida de mulita, donde se observan siete embriones y la cavidad amniótica común (c.am.c.) (Fernández, 1915ª) 
ferroviaria sobre los envíos ayudarían a la confección de los primeros registros y estadísticas sobre la pesca marplatense. Esto último muestra otro aspecto de la relación ciencia y medios de transporte. Otra cuestión que queda por comprobar es la relación entre la extensión de las líneas férreas que se opera en la transición del siglo y el consumo de ciertos productos o la comercialización de animales silvestres en los mercados de Buenos Aires, donde sería factible que esos especimenes fueran estudiados por los zoólogos.

Precisamente, a ese circuito comercial recurriría Fernández. Para sus investigaciones necesitó reunir una considerable cantidad de hembras vivas y preñadas de mulita para seccionar y poder reconstruir a través de cortes en distintos ejemplares el proceso embrionario debido a las dificultades que presentaban estos animales para mantener y reproducir en cautiverio. De hecho, entre 1906 y 1908 tuvo muchas mulitas en cautividad, las cuales fueron muy susceptibles a contraer enfermedades. Varias fueron atacadas por una sarna que en pocos días se extendió a los demás animales de la jaula. ${ }^{3}$ La mayoría de los ejemplares utilizados en sus estudios los adquirió en un comercio local de 'manjares' (Delikatessenhandlung), los cuales, según el dueño del negocio, todos provenían de los alrededores de General La Madrid, en el sudoeste de la provincia de Buenos Aires. Esta localidad era una de las estaciones del Ferrocarril del Sud y distaba cerca de 460km de Buenos Aires y La Plata. Fernández también obtuvo algunos ejemplares que le remitieron colaboradores de dos estancias, una en la provincia de Córdoba (La Cautiva, estación del Ferrocarril Oeste ${ }^{4}$, desde donde en más de una ocasión le enviaron mulitas y principalmente peludos) y otra de la localidad de Marcos Paz, provincia de Buenos Aires. En ambos casos, esos parajes estaban conectados por ferrocarril con Buenos Aires, medio de transporte que seguramente fue utilizado para esos envíos. Cabe señalar que la ciudad de La Plata estaba ubicada a $60 \mathrm{~km}$ y a una hora en tren de la capital federal, existiendo una gran frecuencia de horarios ya que este era el principal medio de transporte de personas y cosas de la capital provincial.

Las hembras de mulita llegaban vivas al laboratorio, donde eran matadas con cloroformo y abiertas para extraer sus vesículas embrionarias (Fernández, 1915a). Estas serían sometidas a varias operaciones técnicas a fin de asegurar su conservación y la visibilidad de su contenido celular. Fernández supervisó todos los detalles de las manipulaciones, los cortes delgados con micrótomo hasta la toma de las microfotografías con la que documentaría sus trabajos. En su primer trabajo sobre estos animales, publicado en la revista alemana Morphologisches Jahrbuch en 1909, analizó sesenta vesículas embriológicas, de las cuales dos representaban estadios previos a la formación de los múltiples embriones. Presentó descripciones detalladas de varias etapas tempranas del desarrollo de la mulita, ilustradas con esquemas, dibujos y microfotografías de muy buena resolución y 'sin retoque', junto con una breve discusión sobre las cuestiones biológicas más importantes conectadas con el fenómeno de la poliembrionía. Fernández fue especialmente afortunado al poder asegurar en un buen estado de preservación dos vesículas embriológicas en las cuales la demarcación de los primordios embriológicos todavía no se había manifestado. Asimismo, encontró que en esos estadios tempranos se producía una inversión de las hojas germinativas como en ciertas especies de roedores. De sus observaciones concluyó que todos los embriones del mismo parto de la mulita, generalmente entre siete y doce, descendían de un sólo huevo y que su separación comenzaba recién después de haberse dividido el embrión único en las 
dos hojas blastodérmicas primitivas. Este fenómeno de producción de crías gemelas o monocigotas de forma regular en cada gestación fue denominado poliembrionía específica.

En 1913 completaría estos estudios con la elaboración de una monografía sobre la embriología y organogénesis de la mulita (Fernández, 1915a) en colaboración con su esposa alemana Kati Marcinowski, también doctorada en la Universidad de Zürich. En otros trabajos, Fernández examinó algunos casos de variaciones individuales en los embriones de la mulita y cuestionó el carácter hereditario de algunas malformaciones. Paralelamente estudió la cuestión de la poliembrionía en otras especies de armadillo. En el caso del 'mataco' y 'pichi-ciego' sólo producían una cría según algunas observaciones e informes, mientras que el peludo tenía generalmente dos crías y por las observaciones en jardines zoológicos, como en el de Berlín, se había considerado que producían gemelos. Sin embargo, Fernández (1915b) encontró que no eran "verdaderos gemelos", ya que en varios casos los embriones eran de diferentes sexos y luego de estudiar su embriología concluyó que cada cría descendía de un huevo diferente y durante su desarrollo se fusionaban los corions. Para estos estudios, logró examinar 120 úteros de peludo, de los cuales sólo 34 tenían embriones, mientras que algunas hembras parieron durante el viaje hasta el laboratorio ya que su período de gestación era mucho más corto que el de la mulita. Para sus investigaciones sobre el peludo no contó con la misma cantidad de ejemplares que de la mulita, un objeto de estudio más fácil de coleccionar gracias a su integración en el consumo urbano.

Entre 1906 y 1909, Fernández pudo disecar 230 úteros de hembras preñadas de mulitas, sesenta de las cuales le fueron remitidas de la Estancia La Cautiva, gran parte sin sufrir daños durante el viaje, y el resto fueron adquiridas en un negocio local, como ya hemos mencionado. De esta forma, logró reunir una serie de cortes representativos de las primeras fases del desarrollo embrionario, algo bastante difícil de obtener por la pequeñez del fénomeno y porque se restringía a un momento particular del año. Las fases más tempranas en el desarrollo serían posibles de observar aproximadamente en los primeros días del mes de junio, lo que implicó realizar varias preparaciones en pocos días. En este caso, la importancia gastronómica de las mulitas y las facilidades en el transporte del campo a la ciudad y al laboratorio fueron circunstancias locales que hicieron posible coordinar el 'tiempo' del investigador y el 'tiempo' del fenómeno. Para los historiadores de las ciencias biológicas, esto último remite al problema de la administración del tiempo (temporalidad), tanto de los organismos como en la forma y organización del trabajo de los investigadores. Cuestiones que por una parte se cruzan con el tema de la visualización de los objetos de investigación y, por otro lado, con los problemas de la prioridad internacional en la publicación y difusión del 'descubrimiento'.

\section{El estudio del armadillo de Tejas}

El problema de los tiempos y las modalidades de transporte de los armadillos para la obtención de los estadios más tempranos del desarrollo estuvo presente en las investigaciones llevadas a cabo por los profesores de la Universidad de Tejas. Horatio Hackett Newman se había doctorado en zoología en la Universidad de Chicago en 1905 y luego de enseñar esa disciplina en la Universidad de Michigan se trasladó a la Universidad de Tejas (Strandskov, 
1958), donde, junto con Patterson, comenzó a estudiar el armadillo de nueve bandas. John Thomas Patterson también se había doctorado en Chicago y hacia 1908 se incorporó al Departamento de Zoología de la Universidad de Tejas. Ambos investigadores se interesaron por la embriología, la determinación del sexo y el estudio de la herencia en esta especie. En 1909 presentaron un informe preliminar sobre la poliembrionía, basándose en varios estadios embriológicos avanzados del armadillo tejano. Al año siguiente publicaron un trabajo donde analizaban setenta vesículas embriológicas, las cuales abarcaban el período comprendido entre la línea primitiva de los embriones hasta el nacimiento y recurrían a la descripción de Fernández para completar una explicación de los primeros estadios antes de la formación de los embriones. Tras dos años de investigaciones, Newman y Patterson no habían podido encontrar las fases más tempranas del desarrollo, una cuestión clave para el estudio de la poliembrionía.

En los años que comenzaron a reunir material para sus investigaciones, el rango de distribución del armadillo por el estado de Tejas se había extendido considerablemente. Cazadores y naturalistas locales habían reportado el hallazgo de ejemplares en nuevas localidades al oeste de Austin, algunas de ellas conectadas por ferrocarril con San Antonio y desde allí con la capital tejana. Newman y Patterson recurrieron a colaboradores y cazadores locales para que les reunieran y enviaran ejemplares de la región semiárida, conocida como Lower Sonoran, y tal vez también de la zona fronteriza con México. Estos investigadores reconocieron que uno de los principales problemas para fijar los estadios más tempranos era el lapso temporal transcurrido durante el transporte de los especimenes preñados hasta su laboratorio en Austin:

During the past two years we have had the opportunity of examining 137 females of the native armadillo, together with a considerable number of males. During the breeding season hunters employed to collect material for us covered a wide range of territory in south-central Texas. These men were frequently obliged to haul the living animals through rough country for distances of fifty miles or more in order to reach an express office whence they could be shipped to our laboratories. As a rule a number of days elapsed between the capture of the annuals and their arrival in Austin. This delay would serve in part to explain our ill success in securing the earliest embryonic stages. In order to obtain a complete series we believe it will be necessary either to breed the animals in captivity or to accompany the hunters on their expeditions so as to lose no time in examining freshly fertilize females. Although we fully expect to secure the earliest stages in the course of time it seems inadvisable for us to postpone the publication of the results thus far obtained ... (Newman, Patterson, 1910, p.363).

En los siguientes años, buscaron cambiar su estrategia para obtener las muestras adecuadas para su estudio. La primera opción, la reproducción en confinamiento, no pareció practicable por el momento, como reconoció Newman. Varios problemas parecían presentarse para la estabilización científica de estos animales en el laboratorio, debido a la baja supervivencia y los cambios de hábitos en cautividad. En ambientes naturales, estos animales eran insectívoros, pero al estar encerrados se volvían carnívoros, mostrando cierta tendencia al canibalismo. Por ejemplo, en encierro o cuando se transportaban en canastas o cajas, el más fuerte mataba y comía al más débil, mientras que las madres devoraban a sus recién nacidos (Newman, 1913). Frente a estos problemas, los autores parecieron optar 

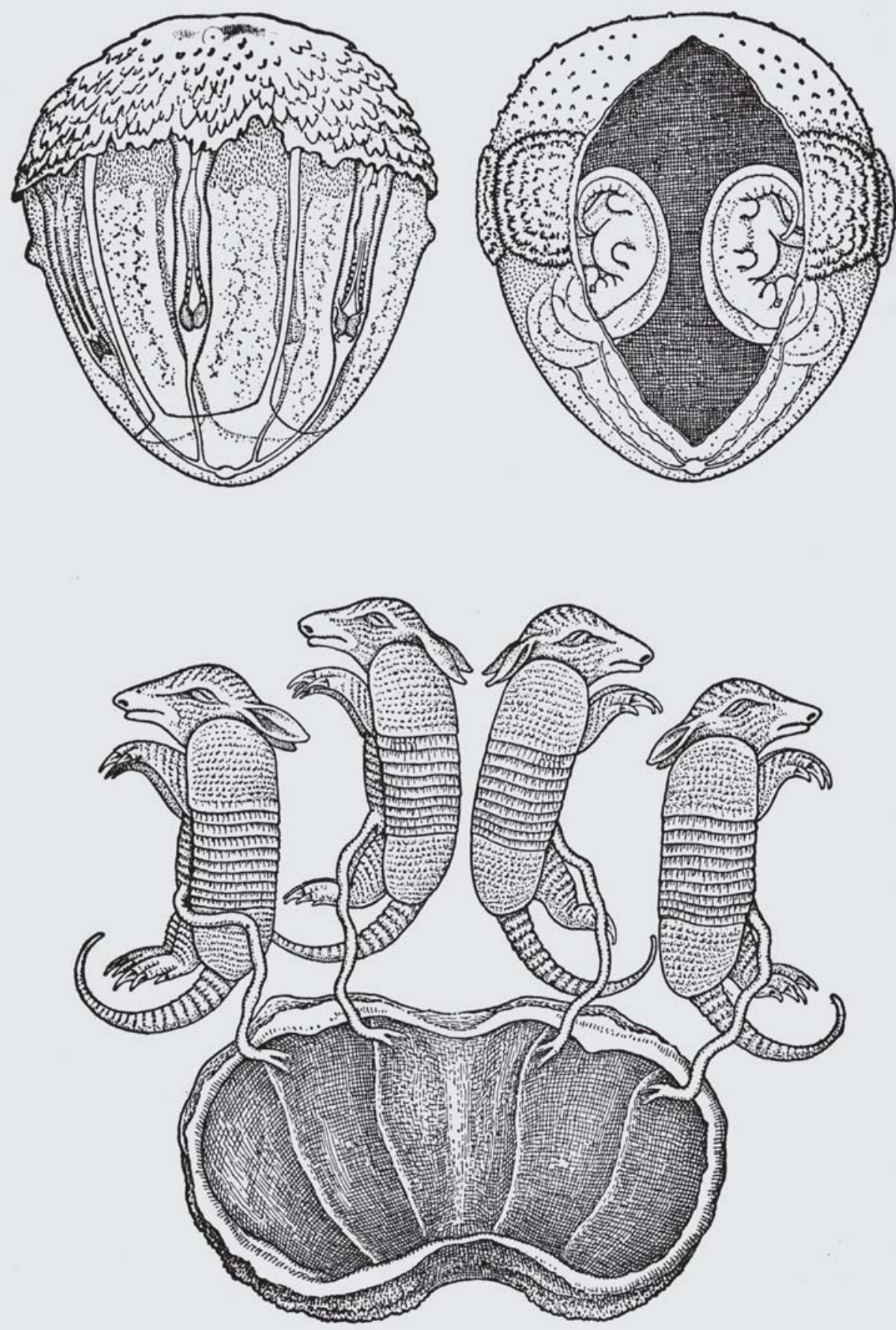

Figura 4 - Tres estados del desarrollo de los cuatro embriones del armadillo texano. En la figura de abajo, la vesícula embriológica ha sido abierta y los fetos removidos (Newman, 1936) 
por la segunda estrategia: trasladarse hasta las poblaciones donde se cazaban y comercializaban armadillos. Newman visitó varias localidades cercanas entre sí y no muy distantes de Austin a donde se podía llegar en ferrocarril a varias de ellas. ${ }^{5}$ Allí pudo obtener una considerable cantidad de hembras preñadas a pesar que la presencia de armadillos en ellas no había sido reportada en el informe sobre la fauna tejana publicada en 1905 por el Bureau of Biological Survey. En algunas, como Boerne, Comfort e Ingram, los animales eran traídos desde una distancia de veinte millas o más por cazadores y granjeros. En las dos primeras ciudades, donde obtuvo cerca de cien y doscientos ejemplares respectivamente, encontró una industria floreciente donde el caparazón del armadillo se utilizaba como materia prima para la confección de cestos que se comercializaban mundialmente como 'curiosidades':

many thousands of the adult animals are slaughtered annually for their armor, which is shaped into basked and sold all over the world as curios. Armadillo hunting has come to be a recreation and a source of additional income for large numbers of young American and Mexican farmers. When they come to town to sell produce and purchase supplies they bring also many armadillo baskets which they have learned to make in an expert fashion and for which there is a ready market. One dealer with whom I am well acquainted claims to have shipped no less than 40.000 baskets during the last six years. At least two other firms have been almost equally active (Newman, 1913, p.515).

Se ha señalado que desde la década de 1890, los armadillos comenzaron a ser promovidos como un 'recuerdo de Tejas' (Texas souvenir), es decir, pocas décadas después de que estos animales se extendieran por el sur de Tejas. Entre los comerciantes de canastas y otros objetos confeccionados con caparazones de armadillos se destacó el inmigrante alemán Charles Apelt, quien exhibió sus productos en la Exposición Internacional de New York en 1902. Desde la década de 1890 hasta su muerte, en 1944, manejó un negocio que alcanzó dimensiones internacionales. La empresa Apelt Armadillo Company, continuada por su familia, operó cerca de Comfort hasta 1971 proveyendo también armadillos vivos a zoológicos e instituciones científicas. Apelt envió animales vivos desde Comfort gracias a las facilidades de transporte provistas por la Southern Pacific Railroad y posteriormente recurriendo a envíos por avión desde San Antonio (Smith, Doughy, 1984). Precisamente en la localidad de Comfort, a pesar de las grandes matanzas de armadillos, Newman no tuvo problemas en obtener en dos semanas cerca de doscientas hembras preñadas para su trabajo. Como reconoció este investigador, "procediendo de acuerdo con los comerciantes de cestas, ha sido posible obtener un abundante material para el estudio embriológico sin aumentar en modo alguno la matanza de esa especie" (Newman, 1922, p.42).

En el otoño de 1910, época del apareamiento del armadillo, lograron reunir numerosas muestras y fijar estadios más tempranos. Newman publicó varios trabajos, algunos en colaboración con Patterson, donde trataron problemas de desarrollo y simetría, citología, determinación del sexo y herencia en el armadillo de Tejas. En 1911 publicaron un estudio estadístico de los embriones de una misma camada, valiéndose de la disposición y el número de placas dentro de las cinturas óseas de los fetos avanzados donde encontraron una semejanza de cerca del $90 \%$ en promedio, y siempre el mismo sexo, entre los embriones de una camada. Una de las conclusiones fue que el sexo era determinado antes de la separación de los embriones. Esto y otros hechos similares parecían justificar la hipótesis de que el sexo estaba determinado en el momento de fertilización. Por otra parte, también trataron 
de apoyar la idea de que cada uno de los cuatro embriones se derivaba de un blastómero del estado de cuatro células del huevo.

Al trasladarse Newman a la Universidad de Chicago se dividieron los estudios. Él se encargó de los problemas vinculados a la herencia y Patterson de completar el estudio del desarrollo embrionario de los armadillos. Patterson dedicó dos temporadas más (1911 y 1912) a fin de obtener las últimas fases de la segmentación del huevo fecundado y las primeras etapas embrionarias. En cada otoño comenzó dos semanas antes a reunir material, encontrando que todas las hembras examinadas durante tres semanas después del 15 de octubre, cuando recogió los primeros estadios, tenían el huevo prácticamente en el mismo estado de desarrollo, por lo que concluyó que existía un 'período de reposo' del blastocito embriológico durante el cual descansaba o se desarrollaba muy lentamente. En 1913, presentó una monografía sobre la serie de estadios muy tempranos, varios más jóvenes que los de Fernández, coincidiendo con este último en que los cuatro embriones del armadillo tejano se formaban de un embrión primario que hasta el estadio de las dos hojas blastodérmicas no permitía descubrir indicio alguno de su división ulterior en varios. Al mismo tiempo, Patterson revisó las teorías que trataban de explicar la poliembrionía, descartando la idea inicial compartida con Newman de que cada embrión derivaba de una de las blastomeras en los que se dividía inicialmente el huevo. Posteriormente, publicaría otros trabajos intentando dar una explicación 'fisiológica' de las causas de la poliembríonia específica, algo que continuaría siendo debatido.

En su monografía de 1913, Patterson también daba cuenta de la metodología ensayada para detectar los estadios más temprano antes de la formación de los múltiples embriones, la forma de cortar el útero, las diferentes sustancias, fijadores y colorantes utilizados en la preservación, entre otras consideraciones. Al analizar algunos estadios, también mencionó ciertos problemas específicos como un error (montaje invertido) en la colocación de un corte en el portaobjetos, pero también en cuanto al transporte de las muestras fijadas y parcialmente endurecidas desde el campo al laboratorio:

I obtained one vesicle which clearly represents a further advance in the progress of vacuolization, and yet one in which the completed stage of the vesicle has not been attained. Unfortunately the specimen became slightly crushed in the course of transportation from the field to the laboratory, after it had been fixed and partially hardened. I therefore deem it unsafe to base any definite conclusions upon its structures; but simply give a photograph of one of the sections ..., which, in a measure at least can be understood after we have considered a normal specimen of a little later stage (Patterson, 1913, p.591).

Como hemos intentado mostrar a través de estos ejemplos, el problema de los tiempos y el transporte de organismos vivos, ejemplares frescos o muestras de los mismos para los estudios histológicos y embriológicos fueron cuestiones importantes con las que se enfrentaron los científicos acá analizados. Aunque en la historiografía de las ciencias biológicas estos son tópicos aún poco trabajados, creemos que el análisis de los mismos puede abrir nuevas e interesantes líneas de indagación en relación a la materialidad de las prácticas científicas. Asimismo, las investigaciones embriológicas sobre la mulita y el armadillo de Tejas permiten examinar otra faceta de la cultura científica: las interrelaciones de la ciencia con el auge comercial o turístico de ciertos productos. 
En ese sentido, las diferencias en el acceso a sus muestras por parte de estos dos grupos de investigadores estuvieron relacionadas no sólo a la disponibilidad de la infraestructura de transporte y comunicación en la región, sino también al circuito mercantil y la forma de comercialización de esos animales en cada contexto social. ${ }^{6}$ En el caso argentino, la carne de mulita era consumida en los centros urbanos, lo que implicaba la necesidad de minimizar los tiempos de transporte entre el campo y la ciudad y de garantizar el buen estado de estos animales enviados vivos ante el riesgo de perder la mercadería o ser decomisada por los controles bromatológicos de los mercados. En cambio, esta necesidad no pareció estar presente en el caso del armadillo de Tejas, cuyo valor comercial estaba dado principalmente por su coraza, muchas veces extraída en los mismos lugares donde eran cazados, lo que generó que los investigadores se transladaran a esas localidades para acortar el tiempo transcurrido entre la captura del animal y su examen científico.

Las estrategias para obtener muestras en el campo y la temporalidad de ciertos fenómenos, los circuitos comerciales en los que participan los objetos u organismos estudiados por los científicos y su trasformación en el laboratorio son cuestiones que permiten vislumbrar diferentes aspectos de cómo trabajaron y qué problemas enfrentaron los científicos en la producción de nuevos conocimientos. Por otra parte, esto también remite a un entramado de relaciones sociales, imperiales, comerciales y sistemas de transporte a través del cual los científicos del laboratorio se conectaron con el campo.

\section{AGRADECIMIENTOS}

Este trabajo forma parte del proyecto PICT ET 3211, dirigido por Irina Podgorny y Arquitecturas de la Ciencia, UAM-Grupo Santander, que dirige Javier Ordóñez. Asimismo se contaron con recursos del proyecto PICT 2005 JOV 33496, a cargo de la autora. Parte de los materiales examinados proceden de la Biblioteca de la Facultad de Ciencias Naturales y Museo de La Plata. Agradezco al personal de la misma, así como al doctor Mariano L. Merino por atender mis consultas y facilitarme los catálogos antiguos de la Sección Mastozoología del Museo de La Plata. También a Irina Podgorny por la lectura del borrador y a Wolfgang Schäffner por las invitaciones a los seminarios y reuniones sobre arquitectura y diseño del saber organizados por la Cátedra Gropius, UBA-DAAD, donde comenzaron a tomar forma algunos de los tópicos aquí analizados.

\section{NOTAS}

${ }^{1}$ Esta cuestión ha sido analizada por Irina Podgorny (e.p.) en relación con los trabajos arqueológicos en el noroeste argentino a fines del siglo XIX y principios del siglo XX.

${ }^{2}$ Macleod (1994) ha mencionado cómo a través de los cables telegráficos submarinos, desde Sydney, se trasmitió en 1884 "monotremes oviparous, ovum meroblasctic" a uno de los miembros de la sección biológica del Congreso de la Asociación Británica Para el Avance de la Ciencia reunido en Montreal, quien informaría sobre ese importante descubrimiento en dicho evento. Así como a través de las redes de la comunicación imperial se informó al mundo que los monotremas ponían huevos con cáscara delgada, tambien "they reminded the world of science of the familiar paradox by which colonial discoveries would become internationally known and rewarded only when recognized by the metropolis" (p.140).

${ }^{3}$ Fernández (1922, p.244-245) reconoció años después que "los desdentados modernos, por lo menos las mulitas, parecen estar expuestas a una o varias variedades de sarna y la afección ofrece en ellas con frecuencia mayor gravedad de la comúnmente observada en animales de denso pelaje... . Entre las muchas mulitas que he tenido en cautividad en los años de 1906 a 1908 se hallaban con frecuencia una que otra tan atacada por una sarna que el caparazón estaba densamente cubierto por costras hasta 
desaparecer en gran parte su escultura característica. En estos casos la afección (cuyo verdadero carácter yo entonces ignoraba) se extendía dentro de pocos días a los demás animales de la jaula".

${ }^{4}$ Es frecuente encontrar en los catálogos de las colecciones antiguas del Museo de La Plata que en la procedencia $\mathrm{u}$ origen de las piezas o ejemplares se registraran localidades, estancias o estaciones de ferrocarril junto con las siglas del ferrocarril al que estaban conectadas. En algunos casos, los nombres de los lugares podían repetirse, pero su vinculación con determinado ferrocarril permitía su identificación.

${ }^{5}$ Las localidades visitadas por Newman fueron: Boerne donde obtuvo cerca de cien ejemplares, en Comfort, doscientos; en Ingram, noventa; en Frederiksberg, cerca de cuarenta; en Kerrville, alrededor de 25 y en Helotes, solo tres. Boerne, Comfort y Kerrville estaban conectadas por el mismo ramal del ferrocarril por el que se podía llegar desde Austin vía la ciudad de San Antonio.

${ }^{6}$ Agradezco esta observación a uno de los evaluadores anónimos de este artículo.

\section{REFERENCIAS}

AZARA, Félix de.

Essais sur l'histoire naturelle des quadrupèdes de la province du Paraguay. París: C. Pougens. 1801.

BENSON, Keith.

Problems of individual development:

Descriptive embryological morphology in

America at the turn of the century. Journal of

the History of Biology, Dordrecht, v.14, n.1, p.115-128. 1981.

BIRABÉN, Max.

Semblanza de Miguel Fernández descubridor de la poliembrionía en los mamíferos. Ciencia e Investigación, Buenos Aires, v.7, p.224-229. 1951.

BOSE, Walter.

Historia de las comunicaciones. In: Academia Nacional de la Historia. Historia argentina contemporánea 1862-1930. Buenos Aires: El Ateneo, v.3. p.575- 655. 1966.

BOWLER, Peter J.

Life's splendid drama: evolutionary biology and the reconstruction of life's ancestry, 1860-1940. Chicago: University of Chicago Press. 1996.

BUGNION, Edouard.

Polyembryony and the determination of sex: resume of observations of P.Marchal. The Smithsonian Report for 1906. Washington, n.1758, p.309-320. 1907.

BURMEISTER, Hermann.

Description physique de la République Argentine: d'aprés des observations personnelles et étrangéres. París: Librairie F. Savy. 1876.

DUGÈS, Alfred.

Sobre el armadillo Cachicana novemcincta.

Memoria de la Sociedad Científica Antonio Alzate, México, v.17, p.35-42. 1902.

DUGÈS, Alfred.

Lettre relative à la placentation des Dasypus novemcinctus. Revue des Travaux Scientifiques, Paris, t.1, p.90. 1881.
CORDERO, Ergasto $\mathrm{H}$.

Miguel Fernández (1882-1950). Ciencia e Investigación, Buenos Aires, v.6, p.335-336. 1950.

FARLEY, John.

Gametes \& spores: ideas about sexual reproduction 1750-1914. Baltimore: The John Hopkins University Press. 1982.

FERNÁNDEZ, Miguel.

Sobre la glándula pelviana y formaciones similares en desdentados recientes y fósiles. Revista del Museo de La Plata, La Plata, t.26, p.212-255. 1922.

FERNÁNDEZ, Miguel.

Die Entwicklung der Mulita. Revista del Museo de La Plata, La Plata, t.21, p.1-519. 1915a.

FERNÁNDEZ, Miguel.

Über einige Entwicklungsstadien des Peludo ('Dasypus villosus') und ihre Beziehung zum Problem der spezifischen Polyembryonie des Genus tatusia. Anatomischer Anzeiger, Jena, v.48, p.305-327. 1915b.

FERNÁNDEZ, Miguel.

Beiträge zur Embryologie der Gürteltiere. 1. Zur Keimblätterinversion und spezifischen Polyembryonie der Mulita ('Tatusia hybrida' Desm.). Morphologisches Jahrbuch, Leipzig, v.39, p.302-333. 1909.

FREEMAN, Michael.

Tracks to new world: railway excavation and the extensión of geological knowledge in mid-nineteenth-century Britain. British Journal for the History of Science, Cambridge, v.34, n.1, p.51-65. 2001.

GARCÍA, Susana.

Miguel Fernández y el proyecto científicoeducativo del Laboratorio de Zoología del Museo de La Plata. Saber y Tiempo, Buenos Aires, v.17, p.99-126. 2004. 
GARCÍA, Susana; PODGORNY, Irina. El sabio tiene una patria. La primera Guerra Mundial y la comunidad científica argentina. Ciencia Hoy, Buenos Aires, v.10, n.55. p.24-34. 2000.

GHISELIN, Michael; GROEBEN, Christiane. Elias Metschnikoff, Anton Dohrn and the Metazoan common ancestor. Journal of the History of Biology, Dordrecht, v.30, p.211-228. 1997.

HOLMBERG, Eduardo.

La fauna de la República Argentina. In: Argentina. Comisión Nacional del Censo. Segundo censo de la República Argentina: Mayo 10 de 1895. t.1. Buenos Aires: Ediciones Talleres de la Penitenciaria nacional. p.477-602. 1898.

HOPWOOD, Nick.

A history of normal plates, tables and stages in vertebrate embryology. The International Journal of Developmental Biology, Bilbao, v.51, p.1-26. 2007.

HOPWOOD, Nick.

Visual standards and disciplinary change: normal plates, tables and stages in embryology. History of Science, Cambridge, v.43, p.239-303. 2005.

HOPWOOD, Nick.

Giving body to embryos: modeling mecahism and microtome in late nineteenth-Century anatomy. Isis, Washington, v.90, p.462-496. 1999.

IHERING, Hermann von.

Über Generationswechsel bei Säugethieren". Archiv für Anatomie und Physiologie.

Physiologische Abteilung, Leipzig, p.443-450. 1886a.

IHERING, Hermann von.

Nachtrag zur Entwicklung von Praopus. Archiv für Anatomie und Physiologie. Physiologische Abteilung, Leipzig, p.541-542. 1886b.

IHERING, Hermann von.

Über die Fortpflanzung der Giirteltiere.

Sitzungsberichte der Königlich Preussischen

Akademie der Wissenchaften, Berlin, v.47, p.105. 1885.

KÖLLIKER, Albert von. Embryologie ou traité complet du développement de l'homme et des animaux supérieurs. Paris: Reinwald LibraireEditeur. Traducción de la décima edición alemana. 1882.

LAMS, Honoré.

Étude de l'œuf de Cobaye aux premiers stades de l'embryogenèse. Archives de Biologie, París, v.28, p.229-323. 1913.
LATOUR, Bruno.

Ciencia en acción. Barcelona: Labor. 1992.

LOPES, Maria Margaret.

Viajando pelo campo e pelas coleções: aspectos de uma controvérsia paleontológica. História, Ciências, Saúde - Manguinhos, Rio de Janeiro, v.8, supl., p.881-897. 2001.

MACLEOD, Roy. Embryology and empire: the Balfour students and the quest for intermediate forms in the laboratory of the Pacific. In: Macleod, R.; Rehbock, P.F. (Ed.). Darwin's laboratory: evolutionary theory and natural history in the Pacific. Honolulu: University of Hawaii Press. p.140-165. 1994.

MAIENSCHEIN, Jane.

What determines sex?: a study of converging approaches, 1880-1916. Isis, Washington, v.75, n.3, p.456-480. 1984.

MILNE-EDWARDS, Alphonse.

Recherches sur les enveloppes foetales du Tatou á neuf bandes. Comptes rendus Hebdomadaires des Séances de l'Académie des Sciences, Paris, v.88, p.406-408. 1879.

MOORE, John A.

Zoology of the Pacific Railroad surveys.

American Zoologist, Utica, v.26, p.331-341. 1986.

MORGAN, Thomas H.

Embriología y genética. Buenos Aires: Losada. 1941.

NEWMAN, Horatio H.

Outlines of general zoölogy. New York: The Macmillan Company. 1936.

NEWMAN, Horatio H.

La biología de los gemelos (mamíferos). Madrid: Calpe. 1922.

NEWMAN, Horatio H.

The natural history of the nine-banded armadillo de Tejas. American Naturalist, Chicago, v.47, n.561, p.513- 546. 1913.

NEWMAN, Horatio H.; PATTERSON, John T. Development of the nine-banded armadillo from the primitive streak stage to birth; with special reference to the question of specific polyembryony. Journal of Morphology, Philadelphia, v.21, p.359-423. 1910.

NYHART, Lynn K.

Biology takes form. Animal morphology and the german universities 1800-1900. Chicago: University of Chicago Press. 1995.

PATTERSON, John Thomas.

Polyembryonic development in Tatusia Novemcincta. Journal of Morphology, Philadelphia, v.24, n. 4, p.559- 683. 1913. 
PODGORNY, Irina

La prueba asesinada: el trabajo de campo y los métodos de registro en la arqueología de los inicios del siglo XX. In: Gorbach, Frida; Beltrán, Carlos López (Ed.). Saberes locales: ensayos sobre historia de la ciencia en América Latina. México: El Colegio de Michoacán. e.p.

PODGORNY, Irina.

Bones and devices in the constitution of paleontology in Argentine at the end of the nineteenth century. Science in Context, Cambridge, v.18, n.2, p.249-283. 2005.

PODGORNY, Irina.

Ser todo y no ser nada: paleontología y trabajo de campo en la Patagonia argentina a fines del siglo XIX. In: Visacovsky, S.; Guber, R. (Comp.). Historia y estilos de trabajo de campo en Argentina. Buenos Aires: Editorial Antropofagia. p.31-77. 2002
PODGORNY, Irina.

El argentino despertar de las faunas y de las gentes prehistóricas: coleccionistas, museos, estudiosos y universidad en la Argentina, 1875-1913. Buenos Aires: Eudeba/Libros del Rojas. 2000.

SMITH, Larry L.; DOUGHTY, Robin W. The amazing armadillo. Austin: University of Tejas Press. 1984.

SORRENSON, Richard.

The ship as a scientific instrument in the eighteenth century.

Osiris, Chicago, v.11, p.221-236. 1996.

STRANDSKOV, Herluf $\mathrm{H}$.

Horatio Hackett Newman. Science,

Washington, v.127, n.3289, p.74. 1958.

VETTER, Jeremy.

Science along the railroad: expanding field work in the US central east. Annals of Science, London, v.61, p.87-211. 2004.

\section{$\rightarrow \rightarrow \rightarrow<<<$}

